\title{
A Novel System for the Identification of Diabetic Retinopathy using Computer Based Statistical Classification
}

\author{
Sunil S S \&Anusuya $\mathbf{S}^{\mathrm{b}}$ \\ aAssociate Professor and Head, Dept. of Computer Science and Engineering, Met's School of Engineering, Mala, Thrissur, \\ India. \\ ${ }^{\mathrm{b}}$ Associate Professor and Head, Dept. of Computer Science and Engineering, Saveetha School of Engineering, Chennai, India.
}

Article History: Received: 10 November 2020; Revised 12 January 2021 Accepted: 27 January 2021; Published online: 5 April 2021

\begin{abstract}
Diabetes Retinopathy (DR) is an eye disorder that affects the human retina due to increased insulin levels in the blood. Early detection and diagnosis of DR is essential in the optimal treatment of diabetic patients. The current research is to develop controls for identifying different characteristics and differences in colour retina and using different classifications. This therapeutic approach describes diabetes recovery from data collected from multiple fields including DRIDB0, DRIDB1, MESSIDOR, STARE and HRF. Here machine learning, neural networks and deep learning algorithms issues are addressed with related topics such as Sensitivity, Precision, Accuracy, Error, Specificity and F1-score, Mathews Correlation Coefficient (MCC) and coefficient of kappa are compared. Finally due to the deep learning strategy the results were more effective compared to other methods. The system can help ophthalmologists, to identify the symptoms of diabetes at an early stage, for better treatment and to improve the quality of life biology.
\end{abstract}

Keywords: Diabetic Retinopathy, machine learning, neural networks, deep learning, Convolutional Neural Networks.

\section{Introduction}

The rapid development of diabetes is one of the biggest concerns for the people in the current healthcare field. Diabetes is a global problem that is rapidly occurring and has a diabetic disorder that causes insufficiency and can lead to many diseases (Lin et al., 2020). DR is the most serious type of diabetes and the leading cause of blindness and blindness in adults. Diabetic retinopathy occurs when there is damage to the arteries of the arteries and often eye damage. Many people have suffered from diabetic retinopathy, as they have no symptoms until the last stage. Therefore, methods of detection and screening are needed very early to prevent vision loss (Melleset al., 2020). Different researchers have proposed multiple classification techniques for fundus images.UsmanAkram et al., 2013, presented precise micro aneurysm identification for early detection of diabetic retinopathy. For classification, Shape, colour, gray level and statistical characteristics are even used. Novel hybrid classifier is being proposed to improve the accuracy. It achieves high sensitivity, specificity, positive prediction, and accuracy. Detailed comparison is performed with prior techniques. Van Grinsv et al., 2013, developed a novel technique, Bag of Visual Word to perform the classification process. The image is divided into patches which extract colour, texture and edges. For the recovery of an image from matching request, a novel approach is used. It is used in query image retrieval and classification of clear lesions. Specificity is increased in the proposed approach and further, this reduces false detection. Pires et al., 2013, developed a solution for the distribution of visual secretions to visualize micro aneurysms. The preprocessing and post processing was performed in retino graphic images. The bags for audio plug-in features meet the low-speed flare visual effects as well as fast-paced images. Max pooling is based on semi-soft coding and is characteristic of midlevel. Operating characteristics of receivers are observed. The suggested classifier is proven to outperform the other classifications. Muhammad Faisal, et al., 2014, designed the filter operations including mathematical morphology, max-tree, and attributes. Jaya et al. developed a fuzzy support vector-machine algorithm to find difficult exudates in the fundus images. Optic disk in the fundus images is extracted by using morphological operations and circular Hough transform. Jagatheesh \& Jenila, 2015 , presented a bag of visual approach words for extracting and classifying DR lesions. The features are extracted by using speeded-up robust features. K-means Clustering, Fisher vector encoding, and max pooling methods are used to construct the visual dictionary. Finally, the DR lesions are identified with SVM classifier. The proposed classifier is noted to be improved in accuracy. There is still a need to improve the sensitivity and specificity values which are considered a limitation in the proposed approach. SagarHonnungar et al., 2016, generated an automatic identification and severity classification of Diabetic Retinopathy by means of machine learning methods in retinal images. In this method involving pre-processing of images, bags of visual words are used for extraction of the element. It categorizes Diabetic Retinopathy's retinal images into different stages by a 
multi-classifier such as LBP (Local Binary Pattern), SURF (Speeded-up Robust Features), and HOG (Histogram of Oriented Gradients). They are featured for constructing a bag of visual words. SVM, random forest in retinal images is used as a multi-class classifier. Prakash et al., 2016, designed a new diagnostic method for the diagnosis of exudates. The K-means are used to detect the optic nerve, and a protective mechanism has been identified to remove the optic nerve. ShrutikaPatil\&ManjiriGogate, 2017, proposed an automatic method for detecting Diabetic Retinopathy. The implemented model consists of pre-processing, extraction of features, and classification stages. The classification technique is K-nearest neighbour (KNN), Gaussian Mixture model (GMM) and SVM. The classifiers KNN and GMM are low in computational complexity. The computational complexity of SVM is proven high. The sensitivity and specificity are calculated and there is noticeable improvement in performance of the proposed technique to find out the exudates in retinal images. AmreenTaj et al., 2017, developed a novel SVM classification scheme. The retinal image is pre-processed by the procedure of median filter and equalization of the adaptive histogram. The pre-processed image is segmented after pre-processing phase. Image segmentation is achieved with k-means clustering algorithm. The exudates are extracted from fundus images, and classification with SVM is used to classify the images. Malathi\&Nedunchelian, 2017, developed a technique for detecting and classifying the various levels of DR. Shrinking edge-mark segmentation technique is used to distinguish the veins and other regions. RSVM (Recursive Support Vector Machine) is used to identify the retinal images and the identification using the proposed scheme is found to be correct. Monzurul Islam et al. 2017, proposed an algorithm that combines image processing with machine learning to study retinal fundus images in diabetic retinopathy. The visual word sack method is used to classify retinal images. The suggested classifier is used to identify fundus images such as lesions, exudates, $94.4 \%$ successful microaneurysms, which is better than other literature approaches. In order to detect the abnormal image various classification concepts (Kashyapet al., 2017) are involved ANN Chandel et al., 2016, KNN (Lupascu et al .,2010), Adaboost technique (Banerjee et al., 2015), Neural Network Classifier Suriyal et al., 2018), Alternating Decision Tree (Roychowdhury et al., 2013). Diabetic Retinopathy (DR) is a common complication of retinal detachment in diabetic patients and causes blindness. The best treatment for retinopathy is early diagnosis and regular monitoring. This takes more time for the ophthalmologists to track or diagnose the disease. If the disease is not diagnosed correctly and at the right time, it may lead to diabetic patients becoming blind. Automatically enables diabetics to identify what is needed to prevent visual acuity. Automatic processing of identification takes less time, and also saves the workload for disease diagnosis. In recent years, automatic detection of diabetic retinopathy using digital fundus imaging has been less effective. The Biomedical has a big boom in the present Technological scenario Suriyal et al., 2018); Hemanth et al., 2020);Zago et al., 2020). The existing techniques lack accuracy, sensitivity and specificity. To overcome the above issues, this research proposes enhanced automatic detection method.

The main objective of the research is

i. To develop deep learning, machine learning and neural network techniques for detection and classification of the DR stages.

ii. To analyze implementation of the proposed CNN classifier with the BOVW classifier and RBF, RNN and MLP networks.

iii. Performance measures are carried out, using a tested on series of digital fundus images to evaluate the metrics, such as Sensitivity, Precision, Accuracy, Error, Specificity and F1-score, Matthews Correlation Coefficient (MCC) and coefficient of kappa, etc.

\section{Methodology}

To detect the diabetic retinopathy stages and performance calculation the following classifiers are used. Here the deep learning and Machine learning classifier is applicable for three cases (normal, Glaucoma and Diabetic Retinopathy) and the neural network is binary classifier it is applicable for 2 cases. The overall flow of proposed work is shown in figure 1 .

- $\quad$ Machine Learning classifier- Bag of visual words

- Neural networks- Recurrent Neural Network, Radial Basis Network and Multi-layer Perceptron Network

- $\quad$ Deep Learning Classifier-Convolution Neural network

\subsection{Machine Learning Classifier}

The word machine learning refers to identifying meaningful anomalies in data automatically. For research fields such as bioinformatics, medicine, and astronomy, machine learning is also commonly used. Training is a very wide subject of course. Therefore, the machine learning area is divided into several sub-areas that deal with different types of learning tasks. Machine learning is a branch of artificial intelligence which is supposed to use 
intelligent software so that machines can perform their jobs competently. The statistical methods of learning form the backbone of intelligent applications used to create machine intelligence.

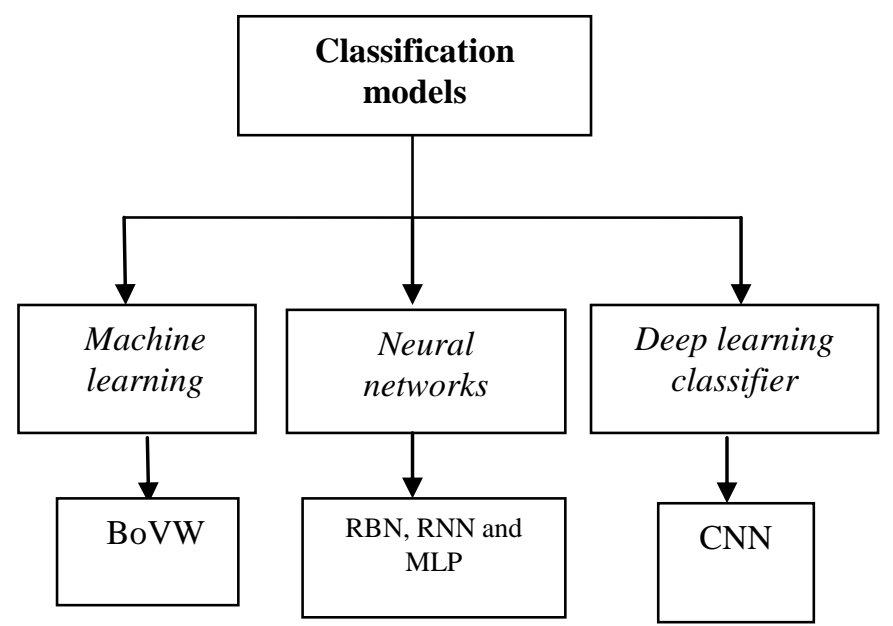

Figure 1.Overall flow of proposed work

\subsubsection{Bag of Visual Words}

Bag of Visual words is a supervised model of learning and an extension to the NLP algorithm. Bag of Words used for classification of images. It's used quite widely aside from CNN. In essence, BOV provides a vocabulary which can best describe the image in terms of extrapolable properties. By generating a bag of visual words it uses the Computer Vision Toolbox ${ }^{\mathrm{TM}}$ functions to define the image categories. The method produces a histogram of occurrences of visual words describing a representation such histograms are utilized to prepare a classifier of the image categories. The steps below explain how to set up your images, develop a bag of visual words then train and apply a classifier for the image type.

It follows 4 simple steps

- $\quad$ Ability of Image features of a defined label

- $\quad$ Development of visual vocabulary by clustering, accompanied by frequency analysis - Classification of generated vocabulary-based images

- $\quad$ Obtain the best class for query image.

Basically, the images are divided into the ones that are training and tested; images are saved for training. A visual dictionary is then created using descriptors to extract representative images of each sentence. Multiclass classifiers are used to learn how to use debug source code with a binary vector support machine. The image on the computer is encoded by a set of tutorials used to extract attributes from the image. A Nearest neighbouring algorithm is used to create a feature histogram in the image. The histogram is the vector element for the image. Finally classification is done.

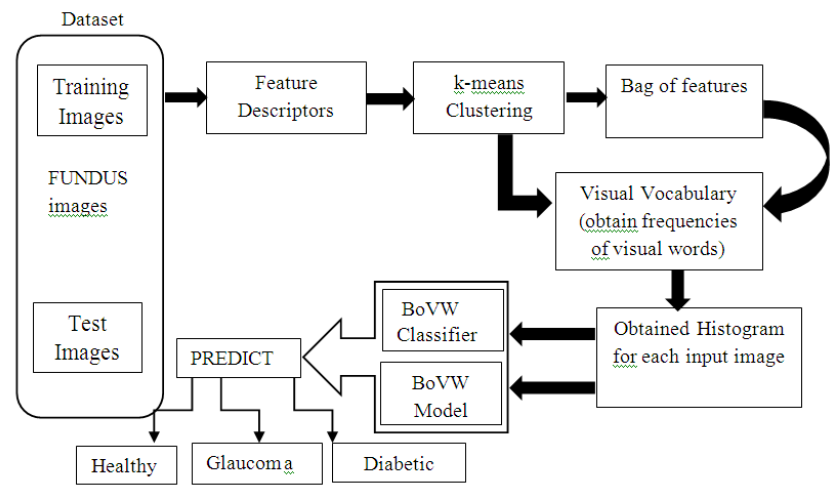

Figure 2. Block diagram of BoVW

Figure 2 shows a basic block diagram of the proposed classification technique. The technique performed by the BoVW classifier is described using blocks.

Steps related to fund image classification: 
1. Find and describe the square image.

2. Assign square image descriptors to a predefined cluster set (a vocabulary) using a vector quantization algorithm.

3. Create a key point bag that counts the total number of drawing boxes assigned to each cluster.

4. Uses a K-means clustering algorithm that treats pockets of salient points as feature vectors and determines the type of eye disease.

\begin{tabular}{|c|}
\hline Training Algorithm \\
\hline $\begin{array}{l}\text { Input ( Collection of Image) Output (K-Clusters, K- } \\
\text { Visual Words) }\end{array}$ \\
\hline $\begin{array}{l}\text { Step 1:Collect set of Images for each class of } \\
\text { Interest(in this paper the class of interest are mild } \\
\text { moderate and severe stages of DR) }\end{array}$ \\
\hline Step 2: Apply BOVW on collected Images. \\
\hline BoVW consist of three main steps: \\
\hline $\begin{array}{l}\text { 1. Extract Key points from images using SIFT } \\
\text { feature detection and description algorithm. }\end{array}$ \\
\hline $\begin{array}{l}\text { 2. Create description for each extracted key } \\
\text { points. }\end{array}$ \\
\hline $\begin{array}{l}\text { 3. Clustering features using K-means Clustering } \\
\text { algorithm(Create Visual Vocabulary using vector } \\
\text { quantization of description space) and save the } \\
\text { resulting "Visual words" }\end{array}$ \\
\hline Testing Algorithm \\
\hline Input (K-Visual Word) Output (Labeled Image) \\
\hline Step 1: Open unlabeled new image \\
\hline $\begin{array}{l}\text { Step 2: Extract and describe features of unlabeled } \\
\text { image using SIFT. }\end{array}$ \\
\hline $\begin{array}{l}\text { Step 3: Extract Visual Words(Centriod) for teasing } \\
\text { image }\end{array}$ \\
\hline $\begin{array}{l}\text { Step 4: Calculate the nearest neighbor using } \\
\text { Euclidean distance between visual words of tested } \\
\text { image and visual words of training images. }\end{array}$ \\
\hline $\begin{array}{l}\text { Step 5: Take the decision: Compare extracted } \\
\text { features of unlabeled image with visual words } \\
\text { extracted in training stage. }\end{array}$ \\
\hline
\end{tabular}

\subsection{Neural Networks}

Neural networks (Bishop 1995) may be able to perform a number of transformation or classification tasks at the same time, while each network usually only performs one. Therefore, in most cases, the network will have a single output variable, although this may result in multiple output units in multi-state classification problems. If you identify a network with multiple output variables, crosstalk can cause problems. The neural methods are:

\subsubsection{Recurrent Neural Network (RNN)}

RNN can be made to a row of any length by repeating the center position and state vector inside ht. reveals hidden status in $\mathrm{h}_{\mathrm{t}}$ time step $\mathrm{t}$ is calculated based on the function of the current icon in previously hidden state $h_{t-1}$.

$$
h_{t}=\left\{\begin{array}{cc}
0 & t=0 \\
\mathrm{f}\left(\mathrm{h}_{\mathrm{t}-1}, \mathrm{x}_{\mathrm{t}}\right) & \text { otherwise }
\end{array}\right\}
$$


The state-to-state transition function $\mathrm{f}$ is often used as an elementary nonlinear composition with both affine transformations is $\mathrm{x}_{\mathrm{t}}$ and $\mathrm{h}_{\mathrm{t}-1}$.

In general, the simplest method of modelling is to enter into a contract to install a veneer conversion vector using RNN and then send the system distribution vector or other transmission service to softmax layer. The problem with the RNN with this shape shift feature is that the vector element of the gradient expands or shrinks rapidly during training along a long line; this discontinuation or gradient omission problem makes successive long-distance correlation studies difficult for the RNN model.

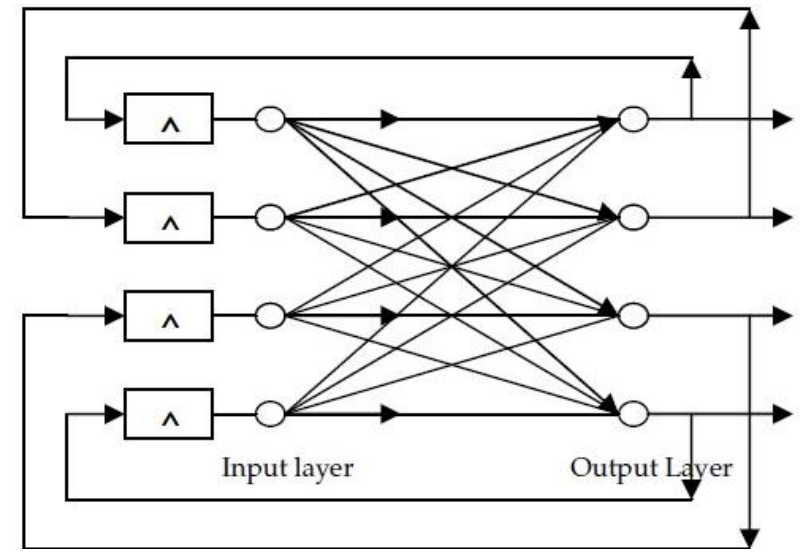

Figure 3.RNN structure

As shown in Figure 1, this recurrent network is referred to as a neural network for re-transmitting communications between individual or additional hidden layers with at least one response cycle. The answer may be a self-replacement, where the output of the neuron returns to its own input. Response cycles often use one unit of delay components, resulting in non-linear dynamic behaviour, with nonlinear units in the neural network. Repeating neural networks have cycle paths (at least one) synaptic connection. A recurrent neural network has a cyclic path (at least one) of synaptic connections.

The value of the model is expressed directly by $u(n)$, and the sample value of the model is expressed in y ( + 1). In this way, the signal vector is added to the perceptron input, which contains the extension data.

Current and past input values, namely $u(n), u(n+1), \ldots . u(n q+1)$, which is exogenous input leaving the network.

The delayed output values, namely, $y(n), y(n-1), \ldots y(n-q+1)$, where the output of model $y(n+1)$ is tracked.

The RNN model dynamic behaviour of is described by

$$
Y(n+1)=F(y(n), \ldots y(n-q+1), u(n), \ldots u(n-q+1))(\text { A. } 2)
$$

Where, $\mathrm{F}$ is a nonlinear function of the arguments.

Algorithm

Step1: Start the program

Step 2: Initialize the input

Step 3: Save Vector input

Step 4: Calculate weight of initial input

Step 5:Calculate value of hidden layer

Step 6:Calculate sigmoid value of $\mathbf{P}$ and $\boldsymbol{\sigma}$

Step 7: Calculate network output

Step 8:If error input=error state vector

Step 9:Yes-repeat step 3

Step 10: No-Stop the procedure 


\subsubsection{Multilayer Perceptron}

Rumelhart and McClelland (1986) discussed most of the most recent books on neural network (Bishop, 1995), it has been the most popular in the network structure used today. Measurements were performed on their valueadded performance and passed this level of operation to generate their value through relocation, and the metrics were divided into open-top processes. Thus, the network has a clear overview of the input models, with the free model, the weight and the initial (non-direct). These may require a simple model with the number of network layers and the number of units in each layer and will determine the system problem. Figure 4 of the multilayer perceptron (MLP) structure includes a description of the hidden number of key issues. Number of units in cells and layers. The calculation of the batting and exit groups is thus determined (as long as access is usable, we will return later, indicating that all will be useful). It is not clear how many units you will need to use. Using a hidden algorithm as long as the numbers are equal to half of the equivalent of the input and output parameters are a good start.

Once you have specified the number of layers and the number of units for each layer, the next step is to determine the weight and the starting point for each connection to reduce network risk. Special training strategies are designed to reduce errors. The data obtained previously was used to correct the weight and the initialization was passed to eliminate this fault. This method is equivalent to the creation of a network structure with training data possible.

The neural network is trained through the network running all of the training scenarios. The network performance is contrasted with the target output, and a specific configuration error can be determined. To get network errors, the variations are combined with the error function. The most commonly used error functions are the total error squares in regression problems, where the individual errors of each unit of output are squared and added up, and the cross entropy function for the highest probability classification.

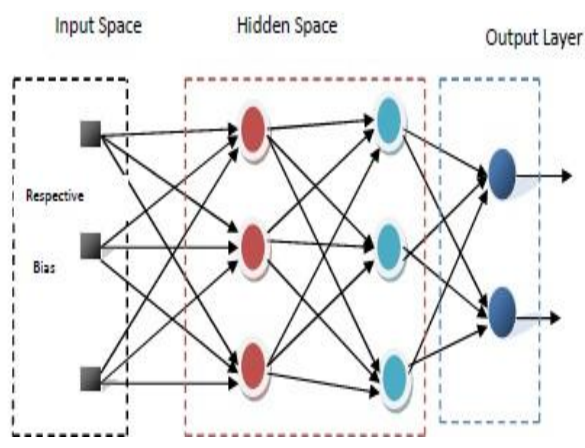

Figure 4.Multilayer Perceptron

A simple perceptron is a single Mc Clock-Pitts neuron trained by the perceptron algorithm.

$O_{x}=g(([w] \cdot[x]+b$

Where $[\mathrm{x}]$ is the input vector, $[\mathrm{w}]$ is the corresponding weight vector, $\mathrm{b}$ is the deviation value and $\mathrm{g}(\mathrm{x})$ is the activation function. Such an arrangement, namely the perceptron, can only classify data that can be separated linearly. In contrast, MLP consists of many layers of neurons. The output equation in MLP with hidden layers is as follows:

$$
O_{x}=\sum_{i=1}^{N} \beta_{i} g\left(\left([w]_{i} \cdot[x]+b_{i}(\mathrm{~B} .2)\right.\right.
$$

Where, $\mathrm{i}$ is the hidden neuron weight value. The process of changing the perceptron weight and bias, or MLP, is called preparation. The perceptron technique (for training simple perceptrons) is to relate the perceptron output to the corresponding target value. The spread of errors is the most common training mode for MLPs. This automated system involves re-deploying error correction across every neuron in the network.

\begin{tabular}{|l|}
\hline Algorithm \\
\hline Step1:Start the program \\
Step2:Increment number of hidden neuron \\
Step3:Initialize MLP \\
\hline
\end{tabular}


Step4:Present data to MLP

Step 5:Train MLP

Step6:Validate error 2-5 times?

Step7:No-repeat step 5

Step8:Yes-save MLP

Step 9:If enough

Step10:No-repeat step 3

Step 11:Yes-continue

Step12:If MLP performance convergence

Step13:No-repeat step2

Step14:Yes-stop procedure

\subsubsection{Radial Basis Functions networks}

A typical RBF network as shown in Figure 5 is a three layered network. It consists of an input layer, an output layer and a closed layer. The input layer receives input from the environment in a multi-dimensional form. Training data should be high. Hidden Layer consists of some locally tuned neurons centered over non-linear, local mapping receptive fields, which is also the same size as the input layer.

The radial base function network can provide a local representation of N-dimensional space. This is achieved through a zone of limited influence of the main function. The parameters of this basic function are given by the reference vector (kernel or prototype) $\mu_{j}$ and the size of the sphere of influence $\sigma_{j}$ : The answer to the main function depends on the Euclidean distance between the input vector $\mathrm{x}$ and the prototype vector $\mu_{j}$ and also depending on the size of the sphere of influence:

$$
\varphi_{j}(x)=\exp \left(\frac{\left\|x-\mu_{j}\right\|^{2}}{2 \sigma_{j}^{2}}\right)(\text { C. } 1)
$$

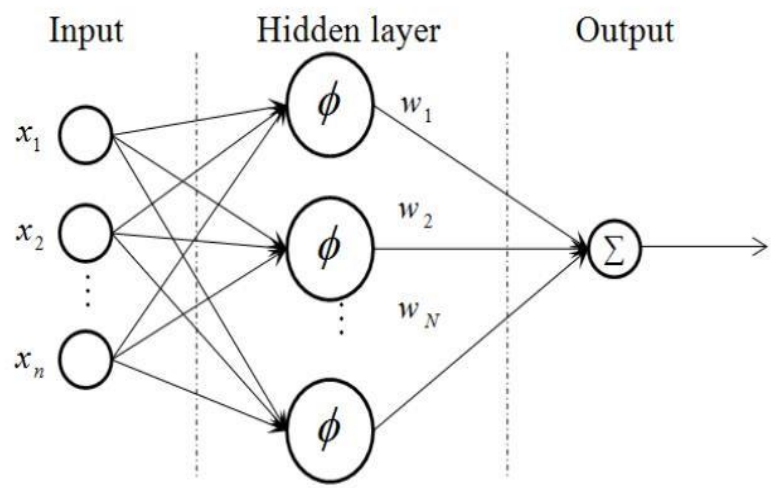

Figure 5.RBF structure

For a given input, the output calculation is given by a limited number of basic functions. Depending on the type of output neuron, the RBF network can be divided into two categories: standardized and non-standardized. In addition, the RBF network can be used in two types of applications: regression and classification.

A standard RBF network consists of an input layer, an output layer, and a hidden layer. The input layer supports input in a multi-dimensional format that is modelled for the environment. Training data should be kept to a minimum. It consists of several neurons from the local hidden layer, centered on a non-linear area of the local mapping and is the same size as the input layer.

Each unit is statistically defined by a function of a radial basis

$$
\varphi_{j}(x)=\varphi\left(\left\|x-x_{j}\right\|\right) \quad j=1,2 \ldots N
$$


Where, $\mathrm{N}$ represents the size of the training sample and $\left\|x-x_{j}\right\|$ is the Euclidean norm of the vector $x-x_{j}$. The jth input data point $x_{j}$. It defines the centre of the RBF, and the vector of the pattern $\mathrm{x}$ is applied to the input layer. Therefore, unlike a multilayer perceptron, the links in the hidden units that connect the source nodes to the nodes are direct weightless links.

\begin{tabular}{|l|}
\hline Algorithm \\
\hline Step1: Select training data and hidden nodes and \\
overlap parameters to be investigated \\
Step2:Identify training and testing data \\
Step3:Perform clustering \\
Step4:Compute network parameter \\
Step5:Compute error for the test set \\
Step6:Compute average error \\
Step7:If investigate all h\&p combination? \\
Step8:Yes continue \\
Step 9:Else repeat step \\
Step 10: Select optimum network as the network with \\
minimum average error and test parameter value \\
Step11:Ready for the use \\
Step12:Stop
\end{tabular}

The output layer consists of fewer numbers or a single computer element is linear, which gives the network response. The Gaussian function is used as a radial base function that defines each computer unit in the hidden layer of the network.

$$
\varphi_{j}(x)=\varphi\left(x-x_{j}\right)=\exp \left(-\frac{1}{2 \sigma_{j}^{2}}\left\|x-x_{j}\right\|^{2}\right), \quad \mathrm{j}=1,2, \ldots, \mathrm{N}
$$

Where $\mathrm{j}$ is the measure of the width of the function of $\mathrm{j}^{\text {th }}$ Gauss function with the center $x_{j}$. All hidden units in Gauss usually receive a specific width, but not always. The parameter that distinguishes one hidden device from another in these situations is the centre $x_{j}$. The reason for Gauss's choice is that it has desirable features based on the radial method of building RBF networks. The approximation function performed by the RBF network structure has a mathematical form

$$
\mathrm{F}(\mathrm{x})=\sum_{j=1}^{K} w_{j} \varphi\left(x, x_{j}\right)(\mathrm{C} .4)
$$

Where, the dimensionality of the input vector is mo and each hidden unit is a function of the radio base. $\left(\mathrm{x}, x_{j}\right)$ where $\mathrm{j}=1,2, \ldots, \mathrm{K}$. The output unit, consisting of a single unit, is characteristic of the weight vector w, the dimensionality also $\mathrm{K}$.

\subsection{Deep Learning}

Deep learning is method for the learning process of artificial neural networks. The neural networks of deep learning are influenced by the workings of the human brain. Deep learning models have many processing units that are called neurons. These neurons do different tasks, such as classification and representation of text. Recently deep learning models show impressive performance in the processing of natural language tasks such as tasks for classifying sentiments including document and sentence classification. The data set also uses deep learning systems to learn complex features. 


\subsubsection{Convolutional Neural Networks (CNNs)}

CNNs are similar to the traditional ANN because they are nerves that adapt to learning. This neuron will still receive input and function (as a scalar product and then unparalleled function), the basis of many ANNs. The whole network will still point to a single reflection function (load) from the input point to the input at the end of the signal line. The final version will include classes related to non-performance, as well as all procedures and procedures designed for ANN to be used regularly. The only difference between CNN and the traditional ANN is that CNN is often used in the field of graphic information. The uniqueness of the image allows it to be incorporated into the architecture, helping the network to match the image performance, as well as the need not to change the format. CNN has 3 types of beds. If applied, this layer creates a pattern on CNN. Figure 6 shows a CNN block diagram.

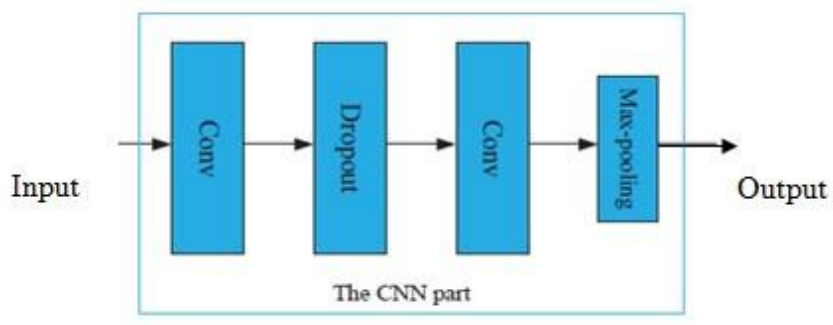

Figure 6. CNN architecture

The main function of the $\mathrm{CNN}$ model is divided into four sections.

i. As seen in other versions of the ANN (Artificial Neural Network), the input element retains the pixel density of the image.

ii. The Assembly system will estimate the nerves connected to the input area by calculating the scales by their weight in the input area. Linear-focused targets (commonly referred to as ReLu) try to add a "smart" one, such as a sigmoid, to the display from the previous layer.

iii. The composition layer will be tested according to the size of the given information, reducing the amount for the task.

iv. All connections will work in the same way as in normal ANN and try to get multiple support systems for distribution. It is said that ReLu can be used to improve the quality of these degrees. CNN can transform a new series from layer to layer by using the folding and sliding method to create a line for the class and the purpose of regression using this simple transition.

\section{Convolutional Layer}

The Convolutional Layer, as the name suggests, plays a key role in the operation of CNN. Layer configuration focuses on the use of learning cores. Such nuclei tend to be small in space dimension, but extend across the input range. When the data reaches a convoluted layer, each filter is returned over the spatial dimension of the input to create a $2 \mathrm{D}$ activation map.

\section{Fully-Connected Layer}

The whole system has a string that connects it to the two nerves next to it, not in their layer. It also seems to be divided into ANN nerves.

\section{Pooling layer}

The purpose of connecting the layers is to gradually reduce the length of the representation, thus increasing the number of inconsistencies and complexities of the structure.
Algorithm
1) Categorize the image dataset
2) Determine the smallest amount of images in a category
3) Use split Each Label method to train the set
4) Notice that each set now has exactly the same number of images 


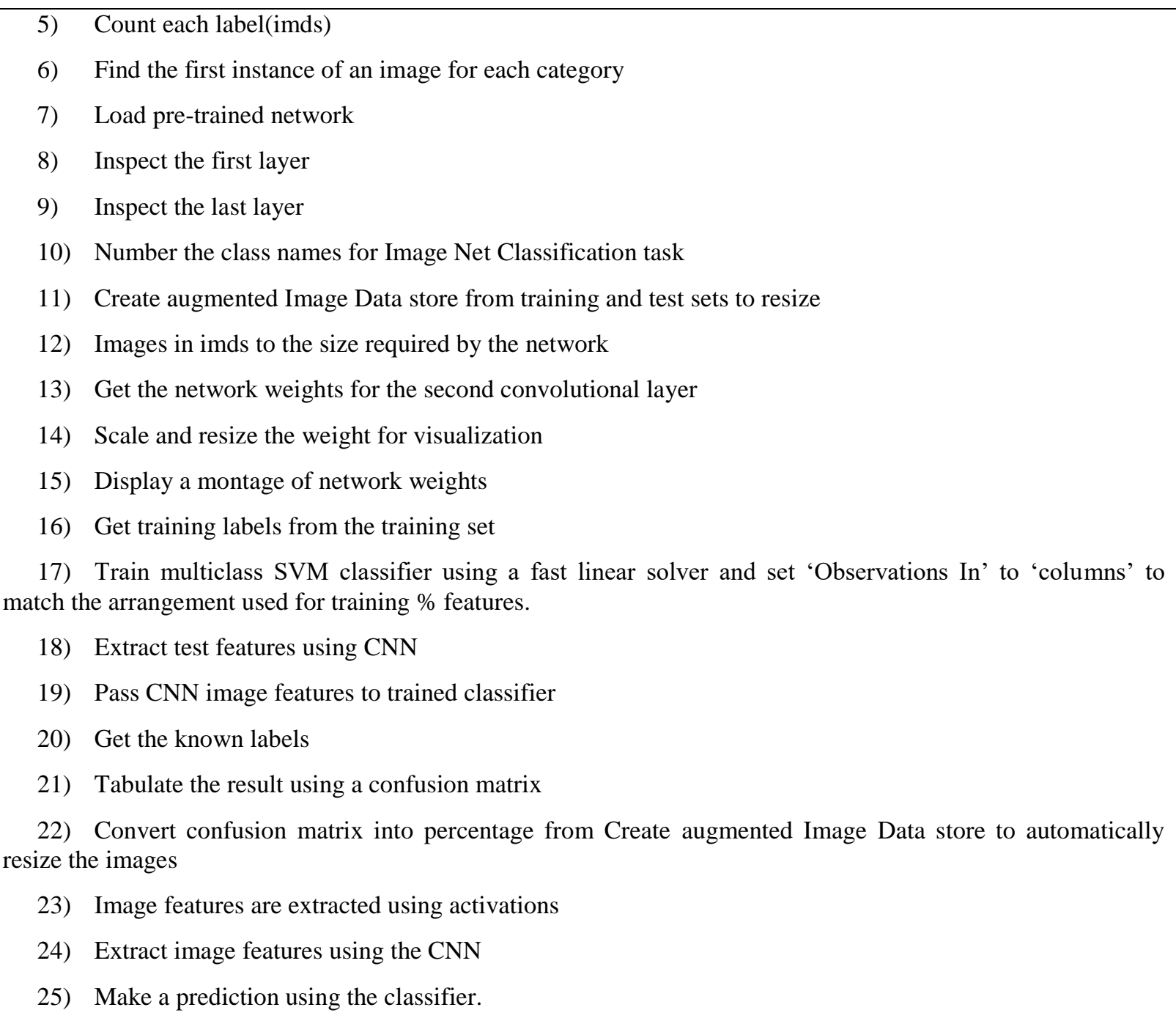

\section{Experimental Result and Discussion}

Retinal images were classified according to the visual classifier, RNN, RBF, MLP and CNN. It classifies retinal images regardless of whether they are normal or abnormal. Services are analyzed for sensitivity, specificity, and accuracy. Finally, several classifiers' performance was compared and it was found that CNN's performance was the best.

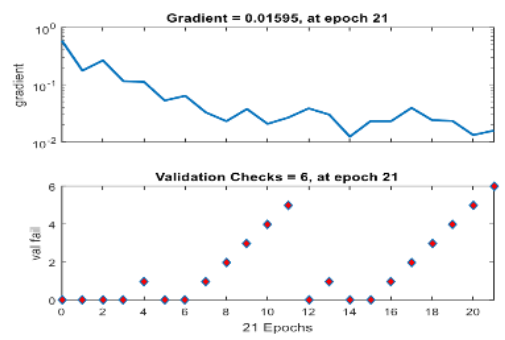

(a)

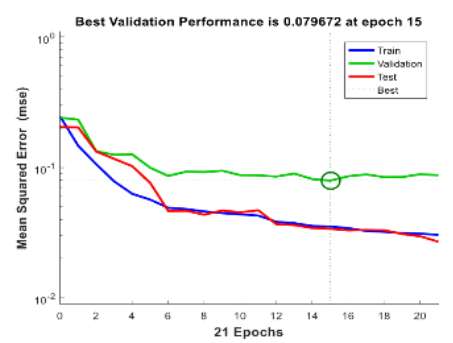

(b) 


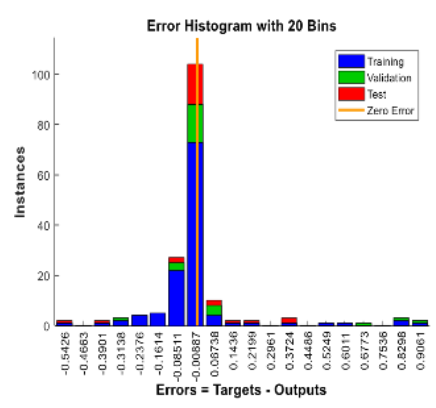

(c)

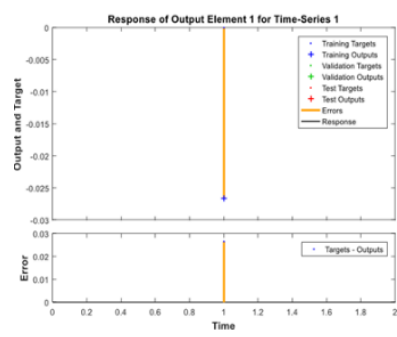

(e)
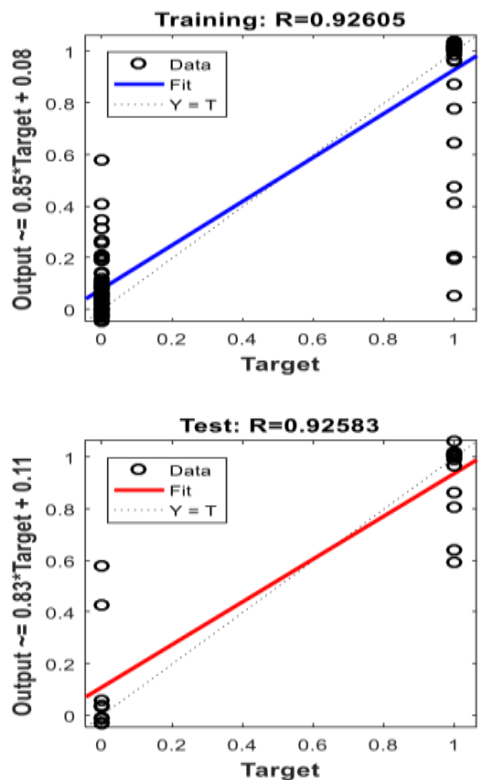

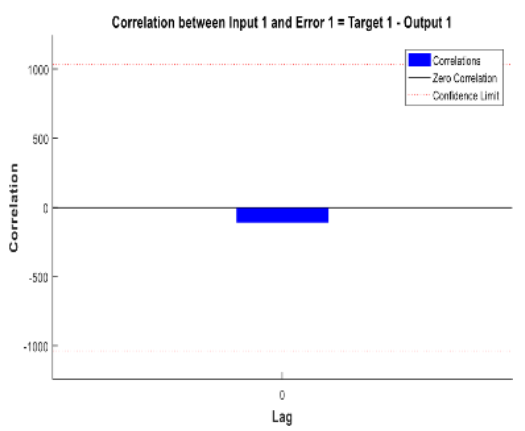

(d)

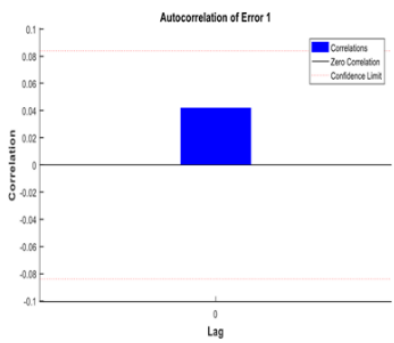

(f)
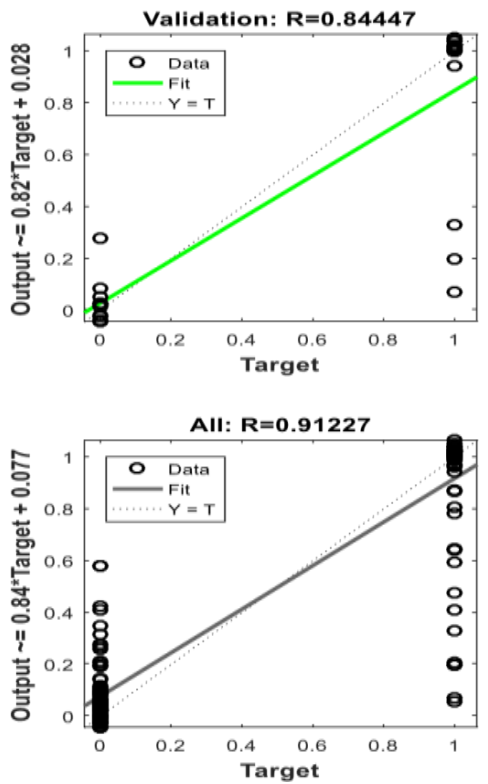

$(\mathrm{g})$

Figure 7. (a) gradient and validation plot (b) performance plot (c) The training plot of error histogram (d) Correlation between target 1 (e) Response of output element 1 for time series (f) autocorrelation of error 1 (g) overall performance of neural system

In RNN classifier the basic training tool of RNN and the performance plot of classifier are shown in Figure 7 \& 8. The Performance, train state, error histogram, autocorrelation of error, Correlation between target 1, Response of output element 1 for time series and receiver operating characteristics are plotted. The performance for each training, validation and test device is shown. Here performance is measured as the root mean square error and recorded in the protocol. With network training, the network retreated rapidly. The confusion matrix shows the percentage of correct and incorrect classifications. The network output is in the range 0 to 1 , here 1 and 0 indicate normal and abnormal patient, respectively. 


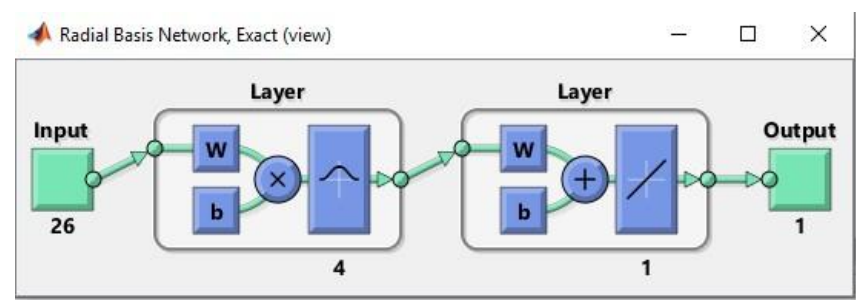

Figure 8. layered architecture of RBN

In RBN classifier the basic training tool of RNN and performance of classifier is shown in Figure $9 \& 10$. Performance characteristics, rail conditions, error histograms and receiver performance are recorded. The performance for each training, validation and test device is shown. Here performance is measured as the root mean square error and recorded in the protocol. With network training, the network retreated rapidly. The confusion matrix shows the percentage of correct and incorrect classifications.

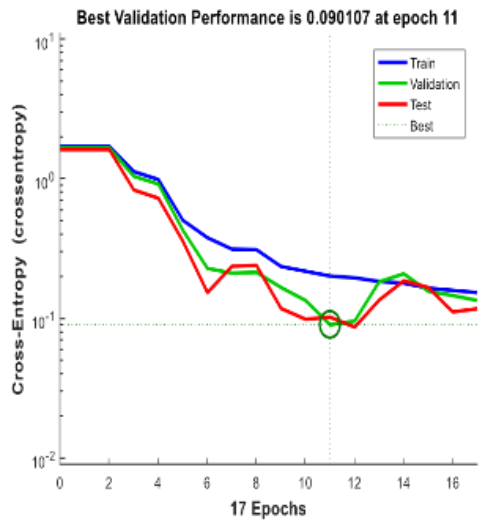

(a)
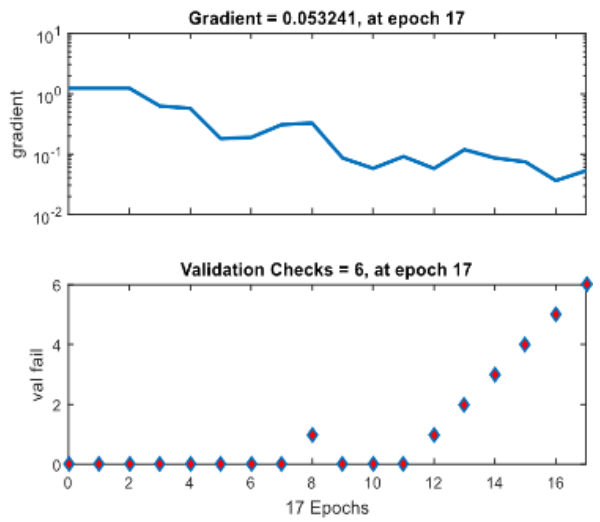

(b)

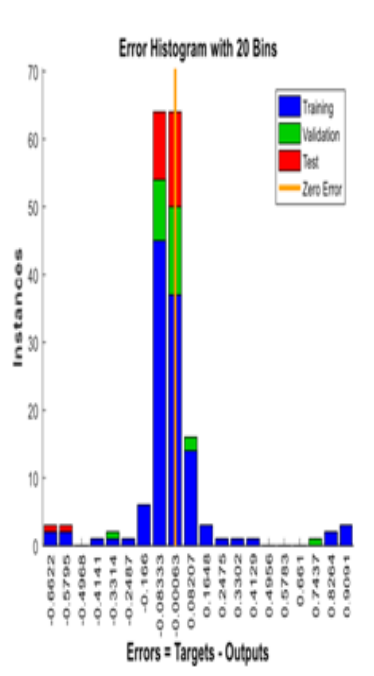

(c)
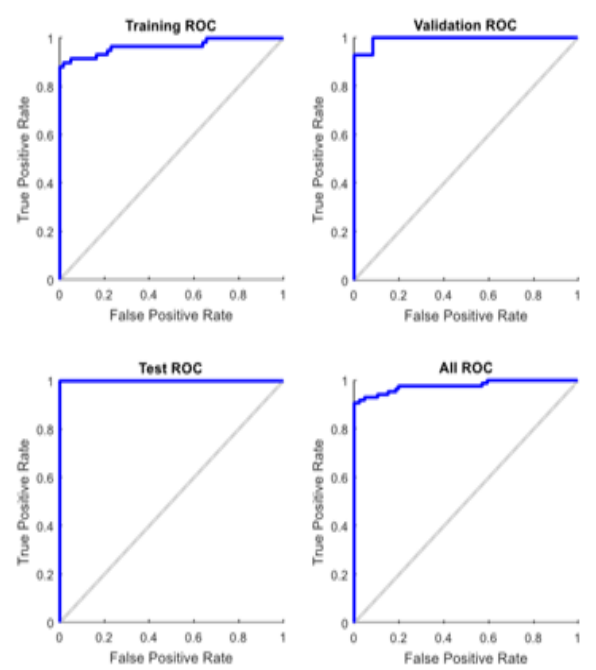

(d)

Figure 9.(a) performance plot (b) gradient and validation plot (c) error histogram plot (d) over all ROC plot of $\mathrm{NN}$

In MLP algorithm the performance is measured in terms of mean squared error shown in figure 11, the average MSE value is 0.4433 . 


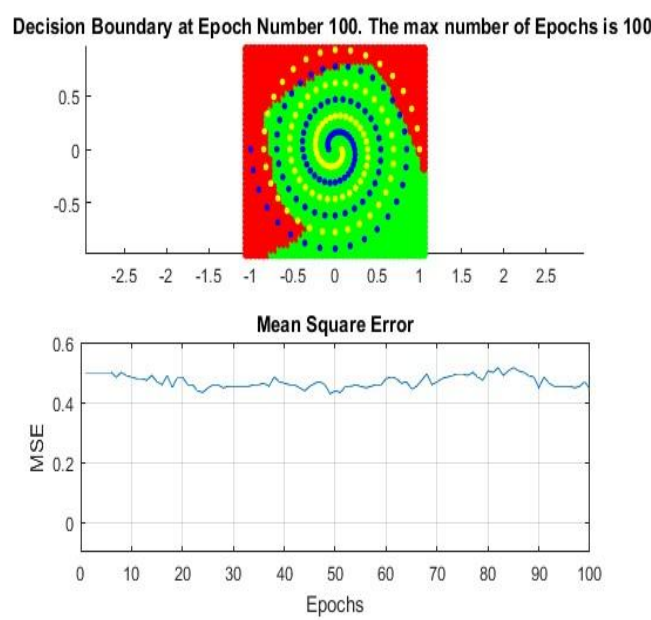

Figure 10. MSE plot of MLP

Figure 12 shows the visual word occurrence of the BoVW classifier. This graphic shows the visual word appearances in the images. The $\mathrm{X}$-axis indicates the visual index of the word and the $\mathrm{y}$-axis indicates the frequency of occurrences in the images, respectively.

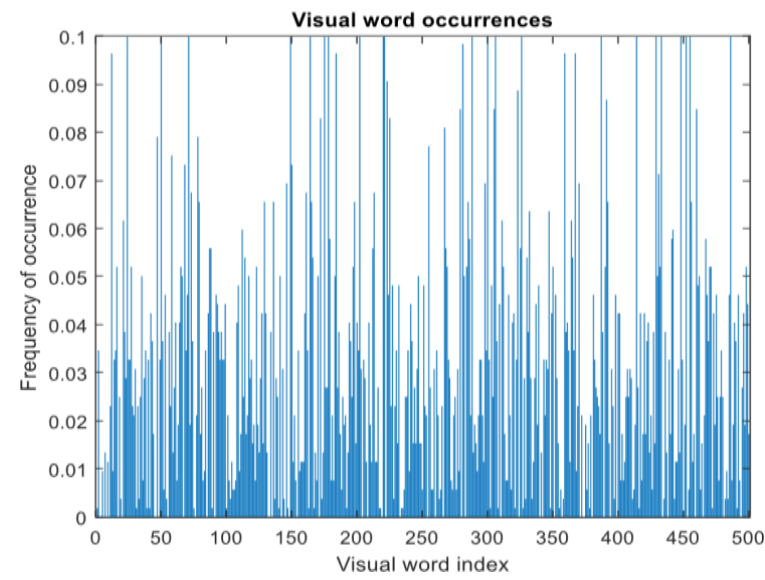

Figure 11.Visual word occurrence of BOVW classifier

CNN's ResNet-50 series has 5 sections, each with the same convolution and block. Each log has three layers and each block has three symbols to achieve. ResNet-50 has over 23 million users. Figure 13(a) and fig 13(b) shows the first section of ResNet and first convolutional layer weights.

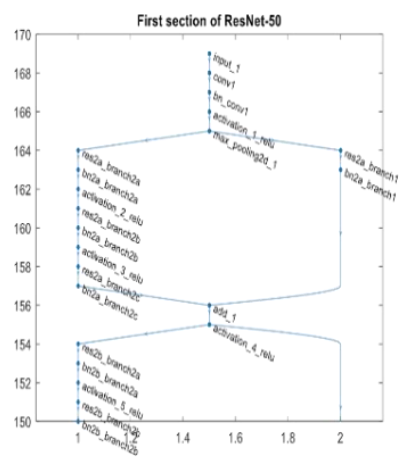

(a) (b)

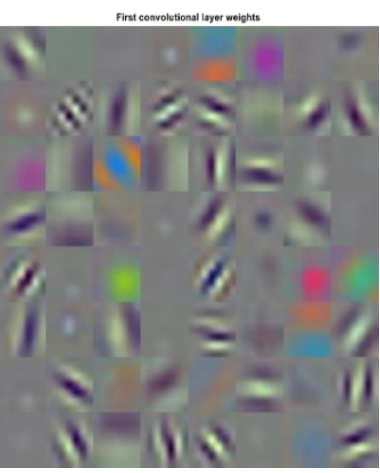

(b)

Figure 12.(a) First Section of Resnet (b) the First Convolution Layer Weights

Confusion matrices are tables that are often used to describe the performance of a data processing system that is characterized by real-world results. This allows understanding how the algorithm works. Each sentence of rhyme represents a class example, while each sentence represents a real class (or vice versa). The precise and confusion matrix are shown in Figure 14. 


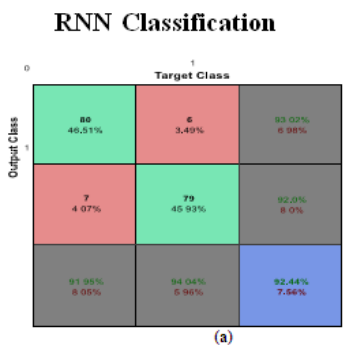

RBN Classification
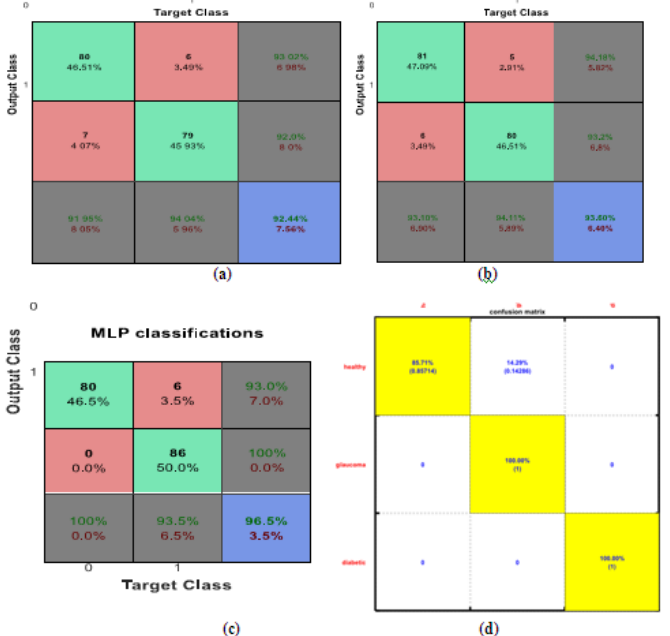

Figure 13.Confusion Matrix of (a) RNN, (b) RBN, (c) MLP, (d) BoVW, (e) CNN

Table 1 shows the performance such as Sensitivity, Precision, Accuracy, Error, Specificity and F1-score, Matthews Correlation Coefficient (MCC) and coefficient of kappa. In this work the accuracy is calculated for every classifier.

Table 1. Performance Comparison of proposed approach

\begin{tabular}{llcccc}
\hline Classifier & RNN & RBN & BOVW & MLP & CNN \\
\hline Accuracy (\%) & 92.44 & 93.6 & 95.24 & 96.5 & 98.33 \\
\hline Error(\%) & 7.68 & 6.40 & 4.76 & 3.5 & 1.59 \\
\hline Sensitivity (\%) & 93.02 & 94.18 & 95.24 & 93 & 98.33 \\
\hline Specificity (\%) & 92.00 & 93.2 & 97.62 & 100 & 100 \\
\hline Precision(\%) & 94.04 & 94.11 & 95.83 & 93.5 & 98.41 \\
\hline F1_score & 0.9249 & 0.9310 & 0.9521 & 0.9643 & 0.9833
\end{tabular}

From the table, F1 is more useful than it is, especially if you have limited class. Truth works well when fake and fake money doesn't cost the same thing. In the third case, the F1 line is 0. Here the MCC is 0.9754 . Normally the MCC has arrange of -1 to 1 where -1 indicates a incorrect classification and 1 indicates the correct classification. The kappa coefficient for the application to be completed is 0.9625 , the kappa measurement is to measure how close the events isolated by the machine to different performance according to the registration data according to the actual ground the accuracy of each difference as measured by the expected accuracy. 9833 . This comparison shows that $\mathrm{CNN}$ classifiers provide the best performance when compared to other classifiers and the classifiers used in the current system produce better performance than other classifiers. Table 2 provides a comparison of performance with existing models. By comparison, the proposed models provide the best results compared to other distribution techniques.

Table 2. Comparison on various existing algorithms

\begin{tabular}{lcrr}
\hline References & Technique & $\begin{array}{c}\text { Existing } \\
\text { Performance }\end{array}$ & $\begin{array}{c}\text { Proposed } \\
\text { Performance }\end{array}$ \\
\hline $\begin{array}{l}\text { Monzurul BoVW } \\
\text { Islam et al. [13] }\end{array}$ & accuracy of $94.4 \%$ & Accuracy of $95.24 \%$ \\
\hline Bhatkar et al. [21] MLP & Specificity of $97 \%$ & Specificity of 100\% \\
\hline Pratt et al. [22] CNN & $\begin{array}{c}\text { sensitivity of } 95 \% \\
\text { and an accuracy } \\
\text { of } 75 \%\end{array}$ & $\begin{array}{r}\text { sensitivity of } 98.33 \% \\
\text { and an accuracy of } \\
98.33 \%\end{array}$ \\
\hline
\end{tabular}




\section{Conclusion}

People impacted by the diabetes require screening to prevent loss of vision. Medical techniques for image processing and sophisticated algorithms are used for detecting the defects present in the retina. The new test automatically detects diabetic retinopathy. Various classification methods are used to classify retinal images and find defects. With the suggested methodology significantly better results are obtained. The values of sensitivity, precision and accuracy are determined from the Matrix of confusion. Finally the CNN classifier is used to identify the stage of Diabetic Retinopathy and its sensitivity, specificity and accuracy, precision, f1-score, MCC and kappa coefficients are measured and compared to other proposed methods. Compared to other methods better results are obtained. The overall accuracy of the proposed classifier is $98.33 \%$. The implemented method is beneficial in detecting early stage Diabetic Retinopathy. The results showed that the algorithms formed can be used to assist an ophthalmologist in classifying retinal images into different stages, thus assisting the ophthalmologist in decisionmaking

\section{References}

AmreenTaj, C, Annapoorna, M, Deepika, KH, KeerthiKumari, BA \&Kanimozhi, S. (2017) 'Detection of Exudates in Retinal Images using Support Vector Machine', International Research Journal of Engineering and Technology (IRJET), vol. 4, no. 5,pp.1847-1852.

Banerjee, Sreeparna, and Amrita Roy Chowdhury. (2015) 'Case based reasoning in the detection of retinal abnormalities using decision trees', Procedia Computer Science 46 402-408.

Bhatkar, AmolPrataprao, and G. U. Kharat. (2015) 'Detection of diabetic retinopathy in retinal images using MLP classifier', In 2015 IEEE International Symposium on Nanoelectronic and Information Systems, pp. 331-335

Chandel, Khushboo, VeenitaKunwar, SaiSabitha, TanupriyaChoudhury, and Saurabh Mukherjee. (2016) 'A comparative study on thyroid disease detection using K-nearest neighbor and Naive Bayes classification techniques', CSI transactions on ICT 4, no. 2-4, 13-319.

Gargeya, Rishab, and Theodore Leng. (2017) 'Automated identification of diabetic retinopathy using deep learning', Ophthalmology 124, no. 7, 962-969.

Hemanth, D. Jude, Omer Deperlioglu, and UtkuKose. (2020) 'An enhanced diabetic retinopathy detection and classification approach using deep convolutional neural network', Neural Computing and Applications 32, no. 3 707-721.

Jagatheesh, C \&Jenila, M. (2015) 'Automatic Detection and Classification of Diabetic Retinopathy Lesion Using Bag of Visual Words Model', International Journal of Scientific and Research Publications, vol. 5, no. 9, pp.1-7.

Kashyap, Nikita, Dharmendra Kumar Singh, and Girish Kumar Singh. (2017) 'Mobile phone based diabetic retinopathy detection system using ANN-DWT.'In 2017 4th IEEE Uttar Pradesh Section International Conference on Electrical, Computer and Electronics (UPCON), pp. 463-467.

Lin, Junnan, Ye Li, and LishiLuo. (2020) 'Effect of Risk Management in Diabetic Retinopathy', International Journal of Diabetes and Endocrinology 5, no. 1: 6.

Ahilan, S. N, et al., "Segmentation by Fractional Order Darwinian Particle Swarm Optimization Based Multilevel Thresholding and Improved Lossless Prediction Based Compression Algorithm for Medical Images". IEEE Access, July 2019, (Volume: 7) PP: 89570-89580.

Malathi, K \&Nedunchelian, R. (2017) 'A recursive support vector machine (RSVM) algorithm to detect and classify Diabetic Retinopathy in fundus retina images”. Biomedical Research, pp. 1-8.

Melles, Ronald B., Carol Conell, Scott W. Siegner, and DariuszTarasewicz. (2020) 'Diabetic retinopathy screening using a virtual reading center', Actadiabetologica 57, no. 2 183-188.

Monzurul Islam, AnhDinh, V \& Khan Wahid, A. (2017) 'Automated Diabetic Retinopathy Detection Using Bag of Words Approach', J. Biomedical Science and Engineering, vol.10, pp.86-96.

Muhammad Faisal, DjokoWahono, Ketut Eddy Purnama, MochammadHariadi\&MauridhiHeryPurnomo. (2014) 'Classification Of Diabetic Retinopathy Patients Using Support Vector Machines (SVM) Based On Digital Retinal Image', Journal of Theoretical and Applied Information Technology, vol. 59, no. 1, pp. 197-204.

Pires, Ramon, Herbert F. Jelinek, Jacques Wainer, Eduardo Valle, and Anderson Rocha. (2014) 'Advancing bagof-visual-words representations for lesion classification in retinal images', PloS one 9, no. 6.

Pires, R, Jelinek, H, Wainer, J, Goldensteins, Valle, E \& Rocha, A. (2013) 'Assessing the Need for Referral in Automatic Diabetic Retinopathy Detection', IEEE Transactions on Biomedical Engineering, vol. 66, no. 12, pp. 3391-3398.

Prakash, NB, Hemalakshmi, GR \& Stella Inba Mary, M. (2016) 'Automated grading of Diabetic Retinopathy stages in fundus images using SVM classifer', Journal of Chemical and Pharmaceutical Research, vol.8, no.1, pp.637-541.

Pratt, Harry, FransCoenen, Deborah M. Broadbent, Simon P. Harding, and YalinZheng. (2016) 'Convolutional neural networks for diabetic retinopathy', Procedia Computer Science 90,200-205. 
Roychowdhury, Sohini, Dara D. Koozekanani, and Keshab K. Parhi. (2013) 'DREAM: diabetic retinopathy analysis using machine learning', IEEE journal of biomedical and health informatics 18, no. 5, 1717-1728.

SagarHonnungar, SanyamMehra\& Samuel Joseph. (2016) 'Diabetic Retinopathy Identification and Severity Classification', CS229, FALL, pp. 1-5.

Shrutika A Patil\&ManjiriGogate. (2017) 'Automatic Screening and Classification using Machine Analysis Technique', International Conference on Emanations in Modern Technology and Engineering (ICEMTE2017) ISSN: 2321-8169, vol. 5, no. 3.

Suriyal, Shorav, Christopher Druzgalski, and Kumar Gautam. (2018) 'Mobile assisted diabetic retinopathy detection using deep neural network', In 2018 Global Medical Engineering Physics Exchanges/Pan American Health Care Exchanges (GMEPE/PAHCE), pp. 1-4.

UsmanAkram, M, Shehzadkhalid\&Shaob Khan, A. (2013) 'Identification and classification of microaneurysms for early detection of diabetic retinopathy', Pattern Recognition, vol. 46, no. 1, pp. 107-116.

Van Grinsven, MJJJP, Chakravarty, A, Sivaswamy, J, Theelen, T, van Ginneken, B, Sanchez, CI. (2013) 'Bag of Words Approach For Discriminating Between Retinal Images containing Exudates Or Drusen’, IEEE 10th International Symposium on Biomedical Imaging.

Zago, Gabriel Tozatto, Rodrigo VarejãoAndreão, Bernadette Dorizzi, and EvandroOttoniTeatiniSalles. (2020) 'Diabetic retinopathy detection using red lesion localization and convolutional neural networks', Computers in biology and medicine 116, 103537.

Zhu, Chengzhang, BeijiZou, Rongchang Zhao, Jinkai Cui, XuanchuDuan, Zailiang Chen, and Yixiong Liang. (2017) 'Retinal vessel segmentation in colour fundus images using extreme learning machine', Computerized Medical Imaging and Graphics 55, 68-77.

Milner Vithayathil, et al., "Neural Proliferation using Brain stimulation Methods Intended for Paediatric Neuropsychiatric Population: A Hypothesis and Theoretical Investigation", Test Engineering and Management, January - February 2020, PP. 9138 - 9151.

S. R. Boselin Prabhu, et al., "Evaluation Strategies for Wireless Ultra-Wideband Communication towards Orthopaedic Surgical Scheme", Journal of Medical Imaging and Health Informatics, December 2018, 8(9):1791-1803(13).

Milner Vithayathil, et al., "Designing and Modelling of a Low-Cost Wireless Telemetry System for Deep Brain Stimulation Studies”, Indian Journal of Science and Technology, 12(8), March 2019. 